Nota / Note

\title{
UNIFORMIDADE DE SISTEMAS DE IRRIGAÇÃO LOCALIZADA: VALIDAÇÃO DE EQUAÇÕES
}

\author{
Gilberto Manilli Favetta ${ }^{1 *}$; Tarlei Arriel Botrel ${ }^{2}$ \\ ${ }^{1}$ Consultor em irrigação, R. Heitor Villa Lobos, 155 - CEP: 13420-130 - Piracicaba, SP. \\ ${ }^{2}$ Depto. de Engenharia Rural - USP/ESALQ, C.P. 9 - CEP: 13418-900 - Piracicaba, SP. \\ *Autor correspondente <gfavetta@iname.com>
}

\begin{abstract}
RESUMO: O coeficiente de uniformidade nos sistemas de irrigação localizada é importante por permitir a avaliação de sistemas ou somente projetados. Quanto aos diferentes métodos aplicáveis, é importante que se defina a correlação entre os mesmos, de forma a permitir a comparação entre os equipamentos. Para avaliação da eficácia de equações de correlação entre os coeficientes de uniformidade de emissão, uniformidade de emissão absoluta e uniformidade estatística, foram comparados os resultados de sua aplicação com valores obtidos pelo uso convencional de cada um desses métodos. A avaliação foi feita a partir de dados obtidos pela aplicação dos três métodos em dois diferentes sistemas de irrigação por microaspersão implantados, tendo sido usado como referência para a avaliação os respectivos coeficientes de uniformidade calculados com base no censo das vazões dos emissores instalados. As baixas diferenças porcentuais entre os valores estimados pelas equações propostas e os obtidos pela aplicação convencional de cada método confirmam a sua validade, permitindo a estimativa da uniformidade de um dos métodos analisados a partir do valor obtido por outro dos métodos estudados.

Palavras-chave: uniformidade de distribuição, irrigação localizada
\end{abstract}

\section{UNIFORMITY OF LOCALIZED IRRIGATION SYSTEMS: VALIDATION OF EQUATIONS}

\begin{abstract}
The localized irrigation systems uniformity coefficient is important to allow evaluation of implanted or just projected systems. With regard to applicable different methods is important to define correlation between them, to allow comparison of evaluated equipments. To evaluate the efficacy of the correlation equations among the coefficients of uniformity of emission, uniformity of absolute emission and statistic uniformity, results of their application were compared to values obtained by conventional measurements. The uniformity coefficients based on the installed emitter flow census were calculated for the evaluated areas and used as a reference. Comparison was made with obtained data by the three methods in two different microsprinkler irrigation systems. Low percent differences between the equation estimated values and those obtained by conventional methods confirmed their validity. This procedure allowed the estimation of the uniformity for one method based on another value estimated by one of the studied methods.
\end{abstract}

Key words: distribution uniformity, localized irrigation

\section{INTRODUÇÃO}

A uniformidade de distribuição é uma informação importante para a avaliação de sistemas de irrigação localizada, tanto na fase de projeto como no acompanhamento do desempenho após a implantação. São disponíveis diferentes métodos para avaliação dessa uniformidade, gerando, no entanto, coeficientes sem base de comparação. Devido ao crescimento da área irrigada por sistemas localizados e a importância da avaliação da sua uniformidade de distribuição, destaca-se a necessidade de correlação entre os diferentes métodos aplicáveis.

Este trabalho procurou avaliar as equações e coeficientes de correlação entre uniformidade de emissão (UE), uniformidade de emissão absoluta $\left(U_{E_{a}}\right)$ e uniformidade estatística $\left(U_{\mathrm{s}}\right)$ obtidas por Favetta et al. (1993), que permitem a comparação de resultados obtidos a partir desses métodos distintos.
O conceito de uniformidade de emissão (UE) foi originalmente apresentado por Keller \& Karmeli (1974), sendo a sua definição baseada na razão entre as vazões mínima e média dos emissores, conforme expresso pela equação 1.

$$
U E=100 \frac{q_{n}}{\bar{q}}
$$

sendo: UE - uniformidade de emissão; $\mathrm{q}_{\mathrm{n}}$ - média das $25 \%$ menores vazões observadas; $\bar{q}^{n}$ - média das vazões observadas.

Para efeito de dimensionamento, conforme observou Bralts (1986), a equação 1 foi posteriormente modificada e redefinida, de forma a incluir o coeficiente de variação de fabricação e o número de emissores por planta, resultando na equação 2. Para efeito de avaliação de campo prevalece a equação 1 . 


$$
U E=100\left(1-1,27 \cdot e^{-0,5} \cdot C V_{f}\right) \frac{q_{n}}{\bar{q}}
$$

sendo: UE - uniformidade de emissão; e - número de emissores por planta; $\mathrm{CV}_{f}$ - coeficiente de variação de fabricação; $q_{n}$ - média das $25 \%$ menores vazões; $\bar{q}$ média das vazões.

Keller \& Karmeli (1974) propuseram uma forma modificada da equação de UE, denominada uniformidade de emissão absoluta $\left(\mathrm{UE}_{\mathrm{a}}\right)$, que inclui as razões das vazões máxima e mínima dos emissores com a média, sendo expressa pela equação 3 .

$$
\mathrm{UE}_{\mathrm{a}}=50\left(\frac{\mathrm{q}_{\mathrm{n}}}{\overline{\mathrm{q}}}+\frac{\overline{\mathrm{q}}}{\mathrm{q}_{\mathrm{x}}}\right)
$$

sendo: $\mathrm{UE}_{\mathrm{a}}$ - uniformidade de emissão absoluta; $\mathrm{q}_{\mathrm{n}}$ média das $25 \%$ menores vazões observadas; $q_{x}$ média das $12,5 \%$ maiores vazões observadas; $\bar{q}$ média das vazões observadas.

Bralts (1986) apresentou os critérios relacionados na TABELA 1 para classificação dos valores de UE e UE

A uniformidade estatística foi primeiramente apresentada por Wilcox \& Swailes (1947) na avaliação de equipamentos de irrigação por aspersão, sendo baseada no coeficiente de variação (CV) da lâmina de água aplicada. De acordo com Bralts et al. (1987), uma abordagem estatística idêntica pode ser feita para os sistemas de irrigação localizada, bastando a substituição das lâminas de água, na conceituação original, pela vazão dos emissores. Esse conceito, aplicado à irrigação localizada, está apresentado nos trabalhos de Bralts (1986), Bralts \& Kesner (1983), Bralts et al. (1981a, b, 1982, 1987) e Benami \& Ofen (1984). Essa metodologia permite a avaliação da

TABELA 1 - Critérios para classificação de UE e UE .

\begin{tabular}{ll}
\hline \multicolumn{1}{c}{ UE e UE } & \multicolumn{1}{c}{ classificação } \\
\hline $90 \%$ ou maior & excelente \\
$80 \%$ a $90 \%$ & bom \\
$70 \%$ a $80 \%$ & regular \\
menor que $70 \%$ & ruim \\
\hline
\end{tabular}

TABELA 2 - Critérios para classificação de $U_{s}$.

\begin{tabular}{ll}
\hline \multicolumn{1}{c}{$U_{s}$} & \multicolumn{1}{c}{ avaliação } \\
\hline $90 \%$ ou maior & excelente \\
$80 \%$ a $90 \%$ & muito bo m \\
$70 \%$ a $80 \%$ & regular \\
$60 \%$ a $70 \%$ & péssimo \\
menor que $60 \%$ & inaceitável \\
\hline
\end{tabular}

uniformidade de distribuição tanto de sistemas implantados como também para efeito de dimensionamento, sendo expressa pela equação 4 . 0 critério para classificação de $U_{s}$ é apresentado na TABELA 2.

$$
U_{s}=100\left(1-C V_{q}\right)=100\left(1-\frac{S_{q}}{\bar{q}}\right)
$$

em que: $U_{s}$ - uniformidade estatística; $\mathrm{CV}_{\mathrm{q}}$ - coeficiente de variação da vazão do emissor; $S_{q}$ - desvio padrão da vazão do emissor; $\bar{q}$ - média das vazões.

Favetta et al. (1993) apresentaram equações de correlação entre os três citados coeficientes de uniformidade de distribuição em irrigação localizada, obtidas através do uso de dados simulados de vazão de emissores. Os resultados mostraram alta correlação entre os coeficientes, sendo apresentadas na TABELA 3 as equações obtidas.

\section{MATERIAL E MÉTODOS}

As equações descritas na TABELA 3 foram avaliadas através de sua aplicação a valores de UE, $U_{E_{a}}$ e $U_{s}$, obtidos da forma convencional. Como referência, foram também calculadas as uniformidades pelos mesmos métodos avaliados mas com base nos dados de censo da vazão dos emissores.

Os dados utilizados foram obtidos em duas áreas irrigadas, cada qual com um tipo de microaspersor: o tipo rotativo, auto regulável, modelo DAN 2001, com vazão nominal de $20 \mathrm{~L} \mathrm{~h}^{-1}$, e o tipo difusor, auto-regulável, modelo DAN 7200, com vazão nominal de $19 \mathrm{~L} \mathrm{~h}^{-1}$. Para avaliação de cada tipo de microaspersor foi adotada uma parcela completa do sistema de irrigação. A cultura irrigada é um pomar de laranjeiras Pera, enxertadas sobre limão Cravo, plantadas em espaçamento $(7 \times 4) \mathrm{m}$ e com um microaspersor por planta. Os microaspersores são espaçados de $4 \mathrm{~m}$ na linha lateral, montada ao longo das linhas de plantio, e colocados na posição central entre duas árvores. A tubulação das linhas laterais é de polietileno de baixa densidade, com diâmetro externo de $16 \mathrm{~mm}$ e interno de $13 \mathrm{~mm}$.

TABELA 3 - Equações de correlação entre entre UE, UE ${ }_{a}$ e $\mathrm{U}_{\mathrm{s}}$ obtidas por regressão linear.

\begin{tabular}{lc}
\hline \multicolumn{1}{c}{ Equação } & $r$ \\
\hline$U E=-37,79586+U_{s}(1,38450)$ & 0,99815 \\
$U E=-10,30951+U_{a}(1,12276)$ & 0,99746 \\
$U E_{a}=9,37365+U E(0,88840)$ & 0,99746 \\
$U_{a}=-24,40025+U_{s}(1,23221)$ & 0,99920 \\
$U_{s}=27,41242+U E(0,72094)$ & 0,99815 \\
$U_{s}=19,85654+E_{a}(0,81090)$ & 0,99920 \\
\hline
\end{tabular}


São descritas na TABELA 4 as características das parcelas e a identificação dos microaspersores em que foi feito o levantamento de vazões.

No procedimento de medida foi feita a numeração dos emissores em ordem crescente, do início para o fim das linhas laterais, sendo a numeração destas em ordem crescente do início para o fim da linha de derivação. Na medida das vazões foram utilizadas provetas de vidro com capacidade de $500 \mathrm{~mL}$, graduadas de $5 \mathrm{em} 5 \mathrm{~mL}$, permitindo leituras a cada $2,5 \mathrm{~mL}$. $\mathrm{O}$ tempo de coleta da vazão foi de 60 segundos, controlado por cronômetro analógico. Durante esse período de 60 segundos, o microaspersor foi inserido dentro da proveta, de forma que não houvesse respingos para fora da mesma. Foram medidas as pressões inicial e final de cada lateral após o término da coleta de vazões de seus emissores.

\section{Uniformidade de Emissão}

UE é dada pela divisão entre as $25 \%$ menores vazões observadas e a média de todas as vazões observadas. Já $U E_{a}$, que é uma variação da uniformidade de emissão, pondera as $25 \%$ menores e as $12,5 \%$ maiores vazões, ambas relacionadas com a média da vazões observadas. Foi utilizada a amostragem padrão para UE e UE ${ }_{a}$, que prevê a medição de vazões em 16 emissores tomados em pontos previamente determinados da área avaliada,

TABELA 4 - Características das parcelas e microaspersores utilizados nos levantamentos.

microaspersor

número de microaspersores modelo quantidade linhas laterais por linha lateral

\begin{tabular}{llll}
\hline DAN 7200 & 336 & 12 & 28 \\
DAN 2001 & 315 & 15 & 21 \\
\hline
\end{tabular}

sendo 4 emissores por lateral, para um total de 4 laterais assim distribuídas: primeira lateral da linha de derivação, lateral a $1 / 3$ do comprimento da linha de derivação, lateral a $2 / 3$ do comprimento da linha de derivação e última lateral. Os emissores de cada lateral foram escolhidos segundo o mesmo critério de distribuição: primeiro emissor, emissor a $1 / 3$ do comprimento da lateral, emissor a $2 / 3$ da lateral e último emissor da lateral. O cálculo de UE e UE $\mathrm{U}_{\mathrm{a}}$ foi feito pelas equações 2 e 3.

\section{Uniformidade Estatística}

$O$ conceito de uniformidade estatística $\left(U_{s}\right)$ é baseado no coeficiente de variação $(\mathrm{CV})$ das vazões dos emissores, que por sua vez é obtido a partir das estimativas da média e do desvio padrão. Uma vez obtido o coeficiente de variação, foi calculado o valor de $U_{s}$, definido pela equação 4

\section{RESULTADOS E DISCUSSÃO}

Os valores estimados pelas equações propostas e os obtidos pela aplicação convencional de cada método são apresentados no TABELA 5, tendo as diferenças entre eles oscilado de $-2,77$ a $0,85 \%$. Fazendo a comparação com os valores de uniformidade calculados a partir do censo das vazões, as diferenças variaram entre $-5,11$ e $0,36 \%$.

Para a aplicação dos valores de uniformidade como referência do que está ocorrendo com os sistemas de irrigação localizada ao longo de seu uso, as diferenças porcentuais encontradas mostram-se muito baixas, confirmando a validade das equações propostas, e viabilizando a comparação entre resultados de uniformidade dos três diferentes métodos, através da estimativa desses coeficientes de uniformidade a partir de um dos mesmos previamente conhecido. O

TABELA 5 - Comparação entre valores de uniformidade observados e valores estimados pelas equações avaliadas.

\begin{tabular}{|c|c|c|c|c|c|c|c|}
\hline \multirow[b]{2}{*}{$\begin{array}{l}\text { modelo de } \\
\text { microaspersor }\end{array}$} & \multirow[b]{2}{*}{$\begin{array}{l}\text { método } \\
\text { estimado }\end{array}$} & \multicolumn{2}{|c|}{ referência } & \multirow[b]{2}{*}{ valor estimado } & \multirow[b]{2}{*}{$\begin{array}{l}\text { valor obtido } \\
\text { pelo censo }\end{array}$} & \multicolumn{2}{|c|}{ diferença (\%) } \\
\hline & & método & $\begin{array}{l}\text { amostra } \\
\text { padrão }\end{array}$ & & & $\begin{array}{l}\text { amostra } \\
\text { padrão }\end{array}$ & censo \\
\hline \multirow[t]{6}{*}{ DAN 2001} & $U_{\mathrm{a}}$ & UE & 90,94 & 90,16 & 93,91 & 0,85 & $-3,27$ \\
\hline & $U_{s}$ & UE & 90,94 & 92,97 & 95,59 & $-2,24$ & $-5,11$ \\
\hline & UE & $\mathrm{UE}_{\mathrm{a}}$ & 91,60 & 92,54 & 94,41 & $-1,02$ & $-3,07$ \\
\hline & $U_{s}$ & $\mathrm{UE}_{\mathrm{a}}$ & 91,60 & 94,13 & 95,59 & $-2,77$ & $-4,36$ \\
\hline & UE & $U_{s}$ & 94,75 & 93,39 & 94,41 & 1,44 & 0,36 \\
\hline & $\mathrm{Ue}_{\mathrm{a}}$ & $U_{s}$ & 94,75 & 92,35 & 93,91 & 2,53 & 0,89 \\
\hline \multirow[t]{6}{*}{ DAN 7200} & $\mathrm{UE}_{\mathrm{a}}$ & UE & 92,47 & 91,52 & 92,86 & 1,02 & $-0,42$ \\
\hline & $U_{s}$ & UE & 92,47 & 94,08 & 94,67 & $-1,74$ & $-2,38$ \\
\hline & UE & $\mathrm{UE}_{\mathrm{a}}$ & 91,80 & 92,76 & 92,98 & $-1,05$ & $-1,29$ \\
\hline & $U_{s}$ & $\mathrm{UE}_{\mathrm{a}}$ & 91,80 & 94,30 & 94,67 & $-2,72$ & $-3,13$ \\
\hline & UE & $U_{s}$ & 96,19 & 95,38 & 92,98 & 0,84 & 3,34 \\
\hline & $\mathrm{UE}_{\mathrm{a}}$ & $U_{s}$ & 96,19 & 94,13 & 92,86 & 2,15 & 3,46 \\
\hline
\end{tabular}


uso dessas equações mostra-se potencialmente interessante na avaliação pós implantação de sistemas de irrigação, devido a escolha dos pontos de coleta para as medições de vazão serem totalmente distintas entre os conceitos de uniformidade de emissão e de uniformidade estatística, e viabilizando a identificação dos diferentes coeficientes de uniformidade a partir de um único método

\section{REFERÊNCIAS BIBLIOGRÁFICAS}

BENAMI, A.; OFEN, A. Irrigation engineering. Haifa: Irrigation Engineering Scientific Publications, 1984. 257p.

BRALTS, V.F. Field performance and evaluation. In: NAKAYAMA, F.S.; BUCKS, D.A. (Ed.) Trickle irrigation for crop production. Amsterdam: Elsevier, 1986. p.216-240. (Development in Agricultural Engineering, 9).

BRALTS, V.F.; KESNER, C.D. Drip irrigation field uniformity estimation. Transactions of the ASAE, v.26, p.1369-1374, 1983.

BRALTS, V.F.; WU, I.P.; GITLIN, H.M. Drip irrigation uniformity considering emitter plugging. Transactions of the ASAE, v.24, p.1234-1240, 1981a.
BRALTS, V.F.; WU, I.P.; GITLIN, H.M. Manufactoring variations and drip irrigation uniformity. Transactions of the ASAE, v.24, p.113-119, 1981b.

BRALTS, V.F.; WU, I.P.; GITLIN, H.M. Emitter plugging and drip irrigation lateral line hydraulics. Transactions of the ASAE, v.25, p.1274-1281, 1982.

BRALTS, V.F.; EDWARD, D.M.; WU, I.P. Drip irrigation design and evaluation based on statistical uniformity concept. In: HILLEL, D. (Ed). Advances in irrigation. Orlando: Academic Press, 1987. v.4, p.67-117.

FAVETTA, G.M.; BOTREL, T.A.; FRIZZONE, J.A. Correlação entre três métodos de estimativa da uniformidade de distribuição em irrigação localizada. Engenharia Rural, v.4, p.117-134, 1993.

KELLER, J.; KARMELI, D. Trickle irrigation design parameters. Transactions of the ASAE, v.17, p.678-684, 1974.

WILCOX, J.C.; SWAILES, G.E. Uniformity of water distribution by some undertree orchard sprinklers. Scientific Agricultural, v.27, p.565-583, 1947.

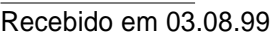

